\title{
Fabric Evolution in Granular Materials Subject to Drained, Strain Controlled Cyclic Loading
}

\author{
C. O’Sullivan* and L. Cui ${ }^{\dagger}$ \\ *Imperial College London, U. K. \\ $\dagger$ University College Dublin, Ireland
}

\begin{abstract}
While there have been many discrete element method (DEM) publications considering the micromechanics of granular materials subject to monotonic loading, studies of the particle-scale material response to cyclic or repeated loading have been comparatively rare. From a geotechnical perspective soil is subjected to repeated loading in a variety of situations. Examples include foundations to railways and roads, foundations to wind turbines, soil adjacent to integral bridges, etc. The work described in this paper extends an earlier study by O'Sullivan et al. [1]. In this earlier study, DEM simulations of strain controlled cyclic triaxial tests were coupled with laboratory experiments to validate a DEM model. The simulations were performed using the axi-symmetric DEM formulation proposed by [2] and a stress controlled membrane algorithm was used to apply forces to balls along the outer vertical boundaries to model the latex membrane used in the laboratory tests. Specimens of uniform spheres and mixtures of sphere sizes were considered in the validation stage of this research.

The earlier study considered strain amplitudes of $1 \%, 0.5 \%$ and $0.1 \%$. In the current study the response is extended to consider the smaller strain amplitude of $0.01 \%$. All of the simulations were carried out in a quasi-static mode and in all cases the maximum stress level mobilized was significantly lower than the peak stress measured in equivalent monotonic physical tests and DEM simulations [2]. In examining the response of the material to the smaller strain amplitude, the macro scale analyses considered the stress strain response and specimen stiffness. At the particle scale, the variation in coordination number and deviator fabric are considered as well as the distribution of the contact forces orientations. The findings may provide insight to the development of continuum constitutive models for cyclic soil response that include fabric parameters [3].
\end{abstract}

Keywords: cyclic loading, fabric tensor, DEM simulations

PACS: $01.30 . \mathrm{Cc}, 02.70 . \mathrm{Bf}, 83.10 . \mathrm{Rs}, 83.10 . \mathrm{Tv}$

\section{INTRODUCTION}

Understanding the response of granular materials to cyclic or repeated loading is important in a wide variety of applications. For example, from a geotechnical perspective pavement sub-bases are subject to repeated loadings as are foundations to wind turbines. Discrete element modelling (DEM) allows engineers to improve their understanding of the evolution of the material fabric during cyclic loading and develop more accurate predictions of long term performance. This paper describes a DEM simulation of a strain controlled cyclic triaxial test with an a cyclic strain amplitude of $0.01 \%$. The simulation approach is firstly outlined, followed by a description of both the macroscale (overall) material response and the evolution of material fabric.

\section{SIMULATION APPROACH}

The approach used to simulate the triaxial tests is described by [2] and [1], for completeness, however, a full description is included here. The current study extends earlier research where DEM simulations were coupled with physical tests ([1]). Consequently, care was taken to simulate the test boundary conditions as accurately as possible. Referring to Figure 1, rigid planar "walls" were used to simulate the top and bottom platens in the triaxial cell. The latex membrane that surrounds the specimen in a conventional triaxial test was modelled by identifying the particles on the outside of the specimen. Then, using a Voronoi diagram, a representative area was associated with each outer particle and a force was applied to the particle, with magnitude equal to the product of the area and the confining pressure. The force was directed to the centre of the specimen. To optimize the computational costs of the simulation, only one-quarter of the test specimen was included in the simulation. Two, vertical radial periodic boundaries were used to maintain a network of particle-particle contacts across the specimen. In this approach, applicable to any axisymmetric DEM analysis, contact between particles close to the periodic boundaries is calculated using an orthogonal rotation tensor.

The "virtual" one-quarter cylindrical specimen generated for the DEM simulations contained 3852 uniform spheres with radii of $2.47 \mathrm{~mm}$. The specimen radius was $50 \mathrm{~mm}$, the height was $200 \mathrm{~mm}$, and the initial void ratio was $\mathrm{e}=0.615$. The Poisson's ratio of the spheres was taken to be 0.28 and the shear modulus was $7.9 \times 10^{10}$ $\mathrm{Pa}$. To reduce the simulation time a scaled density of 


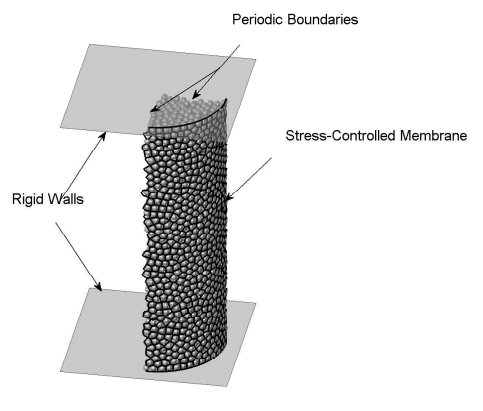

FIGURE 1. Schematic diagram illustrating specimen boundary conditions

$7.8 \times 10^{10} \mathrm{~kg} / \mathrm{m}^{3}$ was used. The confining pressure of $3000 \mathrm{kPa}$ was chosen so that the simulations would be compatible with the simulations considered by [1]. The inter-particle friction coefficient was taken to be 0.096 while a sphere-boundary coefficient 0.228 was used. The simulation loading rate of $0.000333 \mathrm{~mm} / \mathrm{s}$ was chosen to ensure that the specimen response was "quasi-static", i.e. there was negigible difference between the force measured on the top and bottom boundaries in the simulation.

During the simulation, initially the specimen was brought to a stress state close to isotropic. Then the axial strain was increased to $0.01 \%$ (in compression) and subsequently reduced to $0 \%$. This process was repeated 50 times and during these 50 load reversals the macroscopic vertical stress was measured by dividing the vertical force measured on the top and bottom boundaries by the specimen cross-sectional area, while the confining pressure remained constant.

O'Sullivan et al. [1] described validation of the simulation approach by comparing the response of simulations on both the uniform spheres considered here and specimens with a mixture of sphere sizes with physical tests subject to a cyclic strain amplitude of $1 \%$. Considering the 15 loading cycles applied in the physical tests, the DEM simulations captured both the response in the initial load cycle and the subsequent variations.

\section{MACROSCALE RESPONSE}

Figure 2 illustrates the overall stress-strain response for the first, second, tenth and fifthieth cycles of loading. The mobilized stress is illustrated by plotting the deviator stress levels, the deviator stress is the difference between the axial stress and the confining pressure (which remained constant). The oscillations in the response observed in the first cycle are a consequence of the elastic,

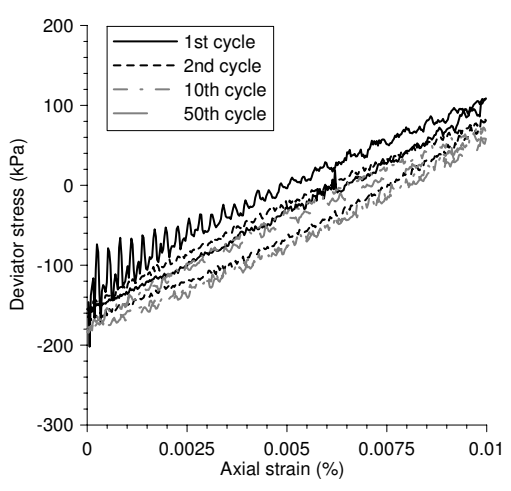

FIGURE 2. Variation in deviator stress as a function of axial strain, cycles 1, 2, 10 and 50 .

non-dissipative contact model used. It is clear from observation of Figure 2 that the deviator stress mobilised at the maximum strain of $0.01 \%$ in the second cycle is lower than the deviator stress mobilised at the maximum axial strain for the first cycle. The variation in mobilised stress values during the subsequent cycles of loading is less marked. However the area enclosed by the hysteresis loop does not vary significantly, indicating that similar amounts of energy are dissipated during each cycle of loading. It appears that as cycling progresses the specimen reaches some type of "equilibrium" state as the difference in response between the tenth and fifthieth cycles is negligible. Note however that the area of the hysteresis loop is finite, representing a dissipation in energy and an inelastic response. As the contact models are elastic, this means that there is either frictional sliding at the contacts or contact conditions are changing during cycling.

Figure 3 illustrates the mobilised deviator stress at the maximum $(0.01 \%)$ and mimimum $(0 \%)$ axial strain values as well as at the intermediate strain level of $0.005 \%$ (during both loading and unloading). All 50 cycles of loading are considered in Figure 3. It is clear that during the initial 5 cycles of loading the decrease in mobilised axial stress values is more marked than in the subsequent 45 cycles. After about 20 cycles, there is still a noticeable variation in the mobilised axial stress values from cycle to cycle, however a general trend of either decreasing or increasing mobilised stresses cannot be observed.

\section{PARTICLE SCALE RESPONSE}

Considering the particle-scale response, two metrics were considered. To quantify fabric a fabric tensor $\left(\Phi_{i j}\right)$ was calculated as:

$$
\Phi_{i j}=\frac{1}{N_{c}} \sum_{k=1}^{N_{c}} n_{i}^{k} n_{j}^{k}
$$




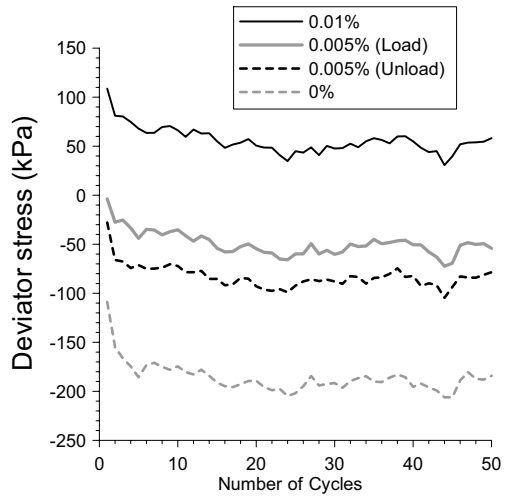

FIGURE 3. Variation in mobilized deviator stress at four levels of axial strain over 50 cycles of loading.

where $N_{c}$ is the total number of contacts and $n_{i}^{k}$ is the component of the unit vector normal to the contact acting in direction $i$. For these three dimensional simulations $i$ and $j$ take values 1,2,3. The anisotropy of the fabric is then taken as the difference between the major principal component of fabric $\left(\Phi_{1}\right)$ and the minor principal component of fabric $\left(\Phi_{3}\right)$. The principal components of fabric are calculated by considering the maximum and minimum eigenvalues of the fabric tensor. The second particle-scale metric considered was the coordination number, $N$. This parameter was calculated as $N=\frac{2 N_{c}}{N_{p}}$, where $N_{p}$ is the number of particles.

Comparing the variation in fabric anisotropy (Figure 4) and coordination number (Figure 5) it can be observed that while it is difficult to draw a direct conclusion regarding variations in specimen anisotropy, the coordination number decreased consistently as cycling continued. Comparing Figures 3 and 5, both the rate of decrease in coordination number and the rate of decrease of mobilised axial stress during the first five cycles of loading are both higher than the subsequent rates of decrease. However, the decrease in coordination number throughout the remainder of the loading cycles does not mirror the decrease in mobilised stress levels.

\section{DISCUSSION}

The results presented in Figures 2, 3, 4, and 5 above can be further explored by comparison with a selected simulation from the study by [1]. The simulation selected from this earlier study had a cyclic stress amplitude of $0.1 \%$ (also strain controlled). For this earlier simulation the macro-scale response is illustrated in Figures 6 and 7 , while the variation in both deviator fabric and coordination number is illustrated in Figures 8 and 9.

Comparing Figures 2 and 6, both the curvature and area of the hysteresis loop are greater for the large cyclic

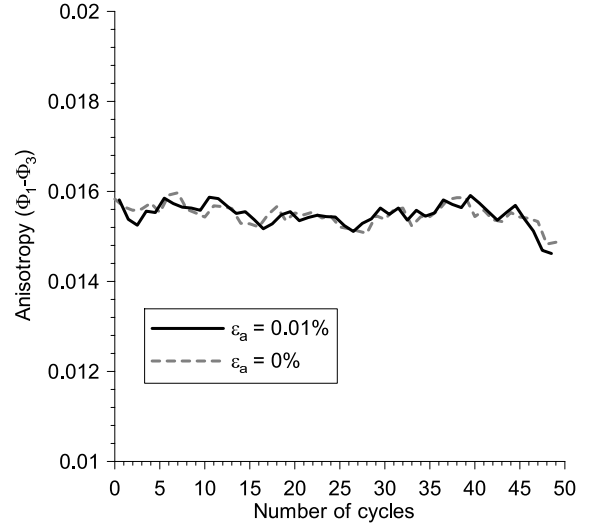

FIGURE 4. Variation in fabric anisotropy over 50 cycles of loading.

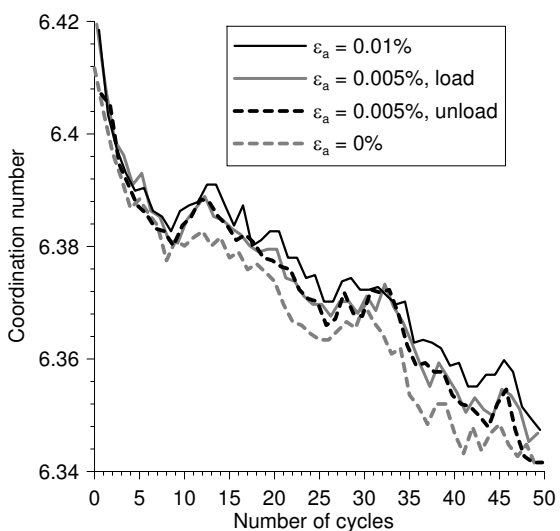

FIGURE 5. Variation in coordination number over 50 cycles of loading.

strain amplitude, reflecting the increased amount of energy dissipated as a greater number of particles loose contact and slide past each other when the imposed macro-scale deformation is larger. The greater rate of decrease in the mobilised axial stresses during the initial cycles of loading is also observed for this larger amplitude of loading (compare Figures 3 and 7).

Considering the particle-scale response, quantified in terms of anisotropy and coordination number, the difference between the two simulations is more marked, in comparison with the differences in macro-scale response. Comparing Figures 4 and 4 , in contrast to the $0.01 \%$ simulation where the variation in anisotropy is negligible, for the $0.1 \%$ strain amplitude simulation there is a consistent trend where the anisotropy at $0 \%$ strain increases, while the anisotropy at the maximum strain level decreases . As before the variation in anisotropy during the initial 5 load cycles is most significant, however during subsequent cyclic there is a noticeable increase in anisotropy at $0 \%$ axial strain and a decrease in anisotropy at $0.1 \%$ 


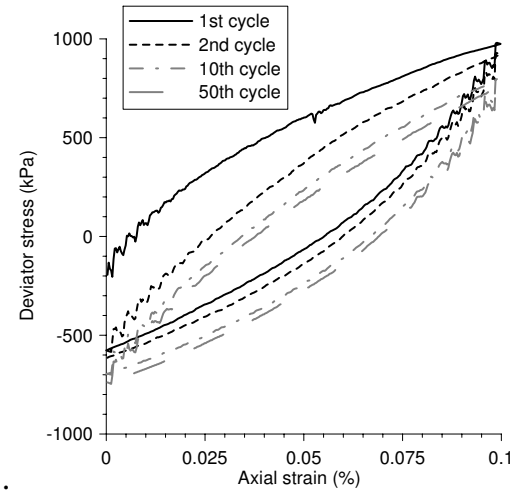

FIGURE 6. Variation in mobilized deviator stress with axial strain for simulation with max. strain $=0.1 \%$.

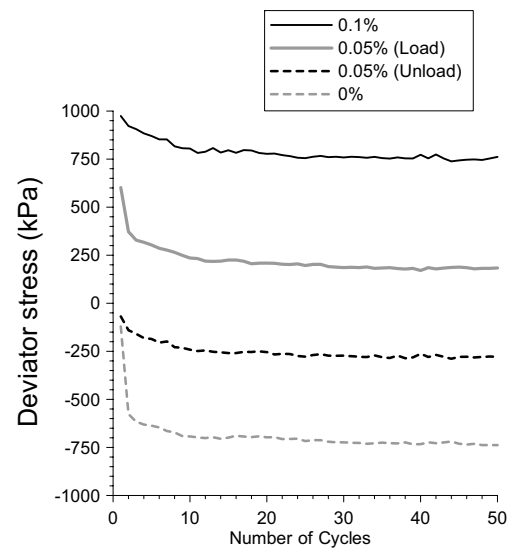

FIGURE 7. Variation in mobilized deviator stress over 50 cycles of loading at selected strain levels for max. strain $=$ $0.1 \%$.

axial strain. In contrast to the $0.01 \%$ simulation the coordination number tends to decrease with the increased amplitude of cyclic straining.

\section{CONCLUSIONS}

This paper extends an earlier coupled numerical - experimental study examining the response of granular materials to drained, strain controlled, quasi-static cyclic loading. In the current study a smaller amplitude of cyclic loading was considered. The variation in macro-scale response, in terms of mobilized axial stresses was less remarkable, than the variation in the granular material packing, quantified in terms of the anisotropy and coordination number. The study illustrates the potential of discrete element modelling to develop our understanding of granular material response under cyclic or repeated loading.

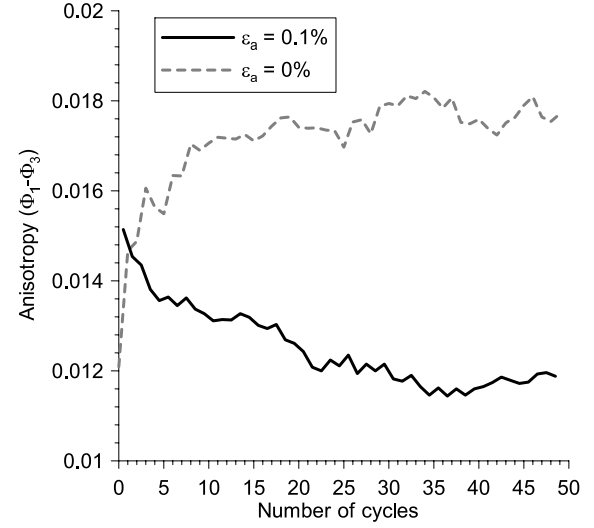

FIGURE 8. Variation in anisotropy over 50 cycles of loading at selected strain levels for max. strain $=0.1 \%$.

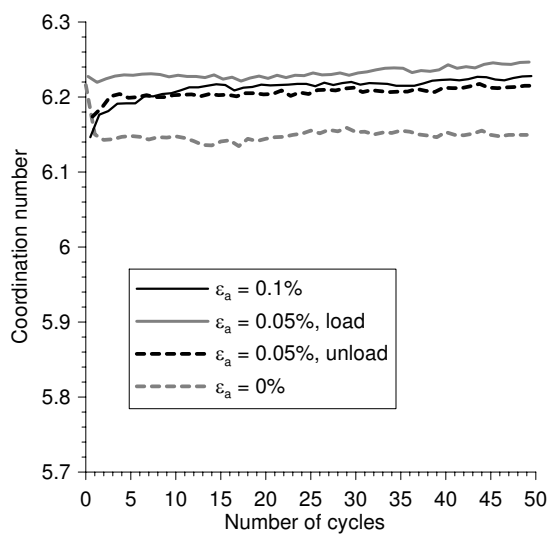

FIGURE 9. Variation in coordination number over 50 cycles of loading at selected strain levels for max. strain $=0.1 \%$.

\section{REFERENCES}

1. C. O'Sullivan, L. Cui, and S. O'Neil, Soils and Foundations 48, 511-530 (2008).

2. L. Cui, C. O'Sullivan, and O'Neill, Géotechnique 57, 831-844 (2007).

3. A. Papadimitriou, and G. Bouckovalas, Soil Dynamics and Earthquake Engineering 22, 191-204 (2002). 